\title{
Violencia de género ante el confinamiento e intervención policial por causa de la covid-19 en Ciudad Juárez, Chihuahua
}

\author{
Gender-Based Violence in the Face of Confinement and Police \\ Intervention Due to COVID-19 in Ciudad Juárez, Chihuahua
}

\section{Violência de gênero em face do confinamento e intervenção policial devido a covid-19 em Ciudad Juárez, Chihuahua}

\author{
Erika Patricia Rojas Gonzalez \\ Universidad Autónoma de Ciudad Juárez, México \\ erika.rojas@uacj.mx \\ https://orcid.org/0000-0002-5290-0110
}

\section{Resumen}

La violencia de género ha aumentado en el mundo en los últimos meses. En México, durante este periodo de aislamiento y de quedarse en casa ante la pandemia del coronavirus, las mujeres corren mayor riesgo de sufrir violencia familiar. En otras palabras, se encuentran en una situación todavía más vulnerable durante esta emergencia sanitaria. El objetivo de esta investigación fue conocer el impacto del confinamiento por la pandemia del SARS-CoV-2 en la dinámica de las familias en Ciudad Juárez, Chihuahua. El enfoque es de carácter documental, por lo que se procedió a la indagación, recolección, organización, análisis e interpretación de información o datos en torno a la violencia contra la mujer en periódicos y bases de datos oficiales. Como parte de los resultados, se encontró que las denuncias por violencia familiar aumentaron cerca de $30 \%$ durante los primeros meses de pandemia. A pesar de este incremento, sin embargo, los diferentes niveles de Gobierno no han tenido una estrategia clara para atenderla.

Palabras clave: COVID-19, intervención, mujeres, violencia familiar. 


\section{Revista Iberoamericana \\ de las Ciencias Sociales y \\ Humanísticas}

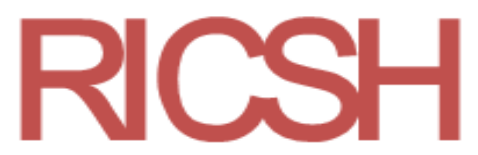

ISSN: $2395-7972$

\section{Abstract}

Gender violence has increased in the world in recent months. In Mexico, during this period of isolation and staying at home in the face of the coronavirus pandemic, women are at greater risk of experiencing family violence. In other words, they are even more vulnerable during this health emergency. The objective of this research was to know the impact of confinement due to the SARS-CoV-2 pandemic on the dynamics of families in Ciudad Juárez, Chihuahua. The approach is documentary in nature, which is why the investigation, collection, organization, analysis and interpretation of information or data on violence against women in official newspapers and databases were carried out. As part of the results, it was found that reports of family violence increased by about $30 \%$ during the first months of the pandemic. Despite this increase, however, the different levels of government have not had a clear strategy to address it.

Keywords: COVID-19, intervention, women, family violence.

\section{Resumo}

A violência de gênero aumentou no mundo nos últimos meses. No México, durante esse período de isolamento e permanência em casa diante da pandemia do coronavírus, as mulheres correm maior risco de violência familiar. Em outras palavras, eles ficam ainda mais vulneráveis durante essa emergência de saúde. O objetivo desta pesquisa foi conhecer o impacto do confinamento devido à pandemia SARS-CoV-2 na dinâmica das famílias em Ciudad Juárez, Chihuahua. A abordagem é de natureza documental, razão pela qual foi realizada a investigação, coleta, organização, análise e interpretação de informações ou dados sobre violência contra a mulher em jornais oficiais e bancos de dados. Como parte dos resultados, constatou-se que os relatos de violência familiar aumentaram cerca de 30\% durante os primeiros meses da pandemia. Apesar desse aumento, no entanto, os diferentes níveis de governo não tiveram uma estratégia clara para enfrentá-lo.

Palavras-chave: COVID-19, intervenção, mulheres, violência familiar.

Fecha Recepción: Julio 2020

Fecha Aceptación: Diciembre 2020 


\section{Revista Iberoamericana \\ de las Ciencias Sociales y \\ Humanísticas}

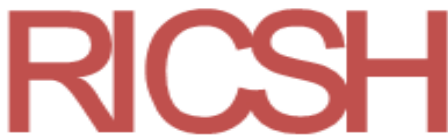

ISSN: $2395-7972$

\section{Introducción}

Según Buchholz (22 de abril de 2020), la violencia de género aumentó en 2020 a raíz del confinamiento por el covid-19. En general, ante situaciones de enfermedad o algún factor que conlleve o que competa a la salud pública se acentúan las desigualdades sociales implicadas en los sistemas socioeconómicos y de salud (Buchholz, 22 de abril de 2020). Sucedió en pandemias anteriores, por ejemplo, la causada por el virus del Ébola, y está sucediendo en la actual, según asientan investigadores de la Universidad de Columbia (Buchholz, 22 de abril de 2020).

"Las normas y los roles de género que relegan a las mujeres al ámbito del trabajo doméstico las sitúan en primera línea en tiempos de crisis, lo que supone un mayor riesgo de exposición; en cambio, se las excluye cuando se desarrollan respuestas de actuación”, indica en una nota de prensa Terry McGovern, autor principal del estudio [publicado en la revista Bioethics] (Buchholz, 22 de abril de 2020, párr. 2).

Teniendo como punto de partida la información anterior, el objetivo de esta investigación fue conocer el impacto del confinamiento por la pandemia de la covid-19 en la dinámica de las familias en Ciudad Juárez, Chihuahua. La preocupación, en términos de supuesto de investigación, es que las posibilidades del aumento de la violencia familiar son muy altas como consecuencia de los espacios reducidos y la poca sensibilización por parte de las autoridades en la ciudad fronteriza en cuestión.

De hecho, así lo deja ver la tendencia internacional. Un claro ejemplo es que, en febrero de 2020, durante la cuarentena aplicada en la provincia de Hubei, China, como medida de protección frente al contagio del virus de la covid-19, se triplicó el número de informes sobre violencia doméstica en la comisaría de la localidad (Buchholz, 22 de abril de 2020).

Asimismo, en España, se ha registrado un incremento. Por ejemplo, en Andalucía se registraron casos de 44 mujeres que fueron víctimas de violencia de género y, por ende, 47 menores a su cargo ingresaron durante el primer mes de confinamiento domiciliario tras el decreto del estado de alarma en este país. ${ }^{1}$ Y en el mismo territorio ibérico, desde la Unidad de Igualdad de la Universidad de Málaga subrayan un aumento en la

\footnotetext{
${ }^{1}$ En respuesta al número creciente de casos de COVID-19 durante la pandemia de 2020, el Gobierno español, en su sesión extraordinaria del sábado 14 de marzo de 2020, declaró el estado de alarma, lo que conllevó como principal medida la imposición de una cuarentena nacional. La medida, que entró en vigor a las 00:00 horas del domingo 15 de marzo, fue una de las medidas de emergencia para reducir el contagio.
} 
probabilidad de sufrir agresiones en el caso de las mujeres por violencia de género a causa de las medidas de emergencia sanitaria ya citadas (Buchholz, 22 de abril de 2020).

\section{Materiales y métodos}

Los paradigmas funcionan como marcos generales en la investigación para comprender los planteamientos y su coherencia con los objetivos. Rodríguez (2013) determina que el paradigma científico "puede entenderse como un conjunto de creencias y actitudes, como una visión del mundo compartida por un grupo de investigadores, y que implica específicamente una metodología determinada en la práctica de la investigación" (p. 23). En este artículo de investigación, se utilizó la metodología cuantitativa, la cual, siguiendo a Tamayo (2014), consiste en el contraste de teorías ya existentes a partir de una serie de hipótesis, y para lo cual es necesario obtener una muestra, ya sea en forma aleatoria o discriminada, pero representativa de una población o fenómeno objeto de estudio (Rodríguez, 2013).

De acuerdo con el objetivo planteado para este trabajo y ante la necesidad de definir el enfoque que ha de guiarlo, se exponen aquí algunos de los atributos del enfoque cuantitativo. Es conocido que para delimitar un paradigma de investigación es necesario tomar en cuenta los ejes epistemológico, ontológico y metodológico, pues, como lo hicieron Guba y Lincoln (2000), estos tres elementos sirven para responder a las preguntas de fondo. En el caso del trabajo que estos dos autores realizaron, ayudó a encuadrar cinco enfoques distintos; de ahí que se pueda afirmar que el acercamiento metodológico cuantitativo sigue la lógica del paradigma.

Según Guba y Lincoln (2000), el paradigma positivista es una visión o sistema de creencias que sirve como guía para que el investigador defina su metodología y sus posicionamientos ontológicos y epistemológicos. En esa línea, King, Keohane y Verna (2000) definen la investigación cuantitativa de la siguiente forma:

El estilo que se sirve de números y métodos estadísticos. Suele basarse en medidas numéricas de ciertos aspectos de los fenómenos; parte de casos concretos para llegar a una descripción general o para comprobar hipótesis causales y busca medidas y análisis que otros investigadores puedan reproducir fácilmente (p.14)

Para este trabajo se llevó a cabo la revisión de fuentes bibliográficas para alcanzar un nivel explicativo, ya que, en él, se consigue determinar las causas del fenómeno y encontrar un mayor entendimiento del fenómeno investigado. Hernández, Fernández y 


\section{Revista Iberoamericana \\ de las Ciencias Sociales y \\ Humanísticas}

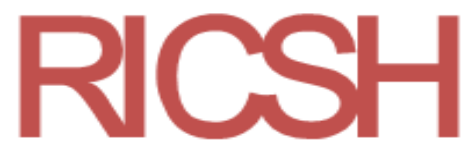

ISSN: 2395 - 7972

Baptista (2010) apuntan que el alcance explicativo "está dirigido a responder por las causas de los eventos y fenómenos físicos o sociales" (p. 85). Además, "como su nombre lo indica, su interés se centra en explicar por qué ocurre un fenómeno y en qué condiciones se manifiesta, o por qué se relacionan dos o más variables " (p. 84). Entonces, este estudio intentará explicar la relación que tiene la cultura escolar y la cultura familiar para lograr la promoción de la salud (Hernández et al., 2010, p. 84).

Por lo anterior, realizamos un análisis científico, un proceso sistemático de indagación, recolección, organización, análisis e interpretación de información o datos en torno al tema de la sobre la violencia contra la mujer en el etapa de confinamiento por el SARS-CoV-2, con el interés de conducir a la construcción de conocimiento nuevos (Morales, 2003).

Cabe señalar que se consultaron fuentes de información primaria solicitadas a la Secretaría de Seguridad Pública Municipal (SSPM) de Ciudad Juárez. Se recurrió, asimismo, a documentos que "son el resultado de otras investigaciones, de reflexiones de teóricos, lo cual representa la base teórica del área objeto de investigación, el conocimiento se construye a partir de su lectura, análisis, reflexión e interpretación de dichos documentos" (Morales, 2003, p. 20).

No se persiguió un significado único, sino que se buscó:

La propia comprensión del fenómeno, la explicación de la realidad a la que se hace referencia, por lo que, la lectura es, en este sentido, un instrumento de descubrimiento, de investigación, de esparcimiento y de aprendizaje; por lo tanto, es fundamental para el desarrollo de los proyectos (Morales, 2003, pp. 22).

\section{Resultados}

Ciudad Juárez no es la excepción respecto al aumento de denuncias por violencia de género. Durante este periodo de aislamiento y de quedarse en casa ante la pandemia del coronavirus, las mujeres han padecido mayor riesgo de sufrir violencia familiar. En otras palabras, se encuentran en una situación todavía más vulnerable durante esta emergencia sanitaria.

Verónica Corchado Espinoza, directora general del Instituto Municipal de las Mujeres de Ciudad Juárez (IMM), mencionó que, de acuerdo con información periodística, en países como China, Italia y España aumentó casi $30 \%$ el número de denuncias por violencia familiar debido al aislamiento por el coronavirus, "lo cual nos 


\section{Revista Iberoamericana \\ de las Ciencias Sociales y \\ Humanísticas}

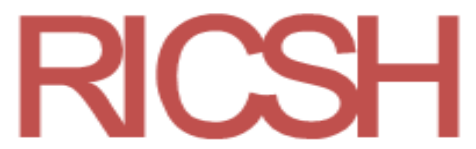

ISSN: $2395-7972$

pone en alerta para darle seguimiento a este tema de manera muy puntual en Juárez con la Unidad Especializada en Violencia Doméstica (Unevid)" (H. Ayuntamiento de Juárez, 3 de abril de 2020).

Continuando con datos periodísticos, los meses con mayor número de llamados de auxilio fueron marzo y abril, según los reportes recibidos en $\mathrm{C} 4$ a través del número de emergencia 911: la barrera de las 2000 llamadas por mes fue superada (Miranda, 21 de mayo de 2020).

Asimismo, siguiendo la información recabada mes tras mes desde finales del 2019 hasta abril del 2020, en diciembre fueron registradas 2210 llamadas de violencia, para enero la cifra bajó a 2069 llamadas, en febrero se incrementó otra vez a 2137, en marzo se llegó a 2402 y abril cerró con 2392.

En promedio, para enero del 2020 se atendieron cerca de 67 llamados por día; para febrero fueron 76 diarias; en marzo, 77 y en abril se mantuvo la estadística de 80 denuncias recibidas día a día al 911.

A continuación, se muestra una gráfica con los índices de denuncias por violencia familiar en el estado de Chihuahua.

Figura 1. Municipios con mayor número de denuncias

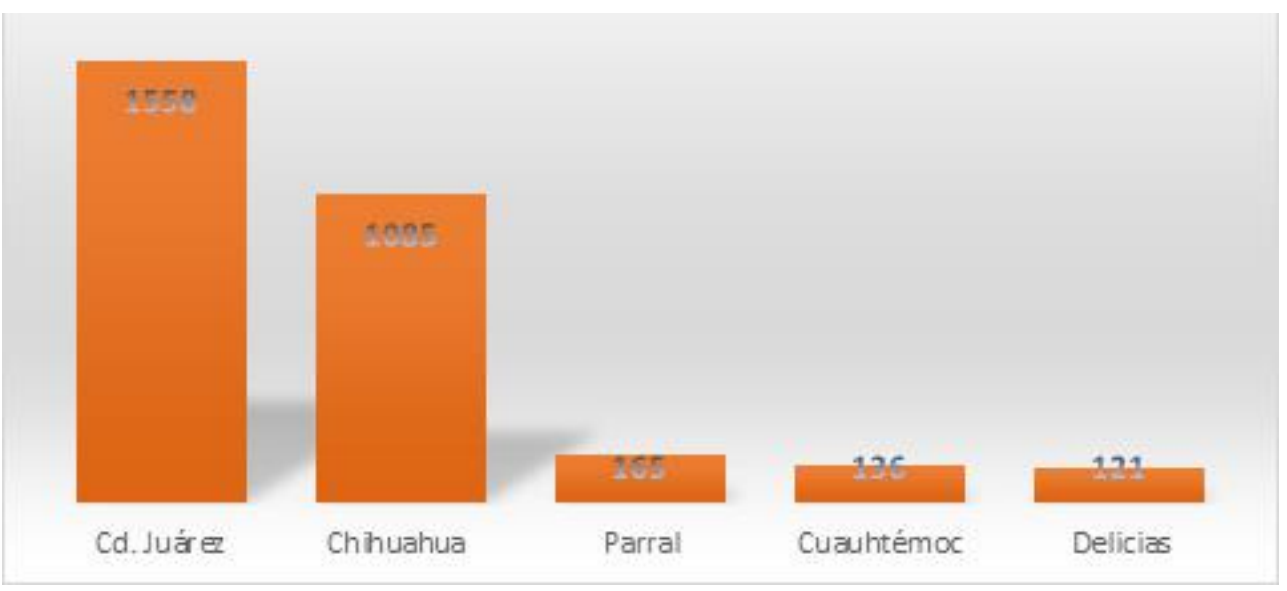

Fuente: Fiscalía Especializada de la Mujer Chihuahua (2020)

En esta gráfica se visualiza que Ciudad Juárez tiene el mayor índice de denuncias del 1 enero al 15 de abril. Entendemos que el confinamiento empezó desde mediados de marzo en México y se ha extendido por lo menos hasta el 1 de diciembre.

Para explicar la crisis y la violencia de género debemos partir de un enfoque sociocultural. Para Ritzer (2003), hasta hace poco lo masculino había sido tomado como punto de referencia para los estudios de naturaleza social por arriba de lo femenino. Una de las causas de tal hecho es que los valores y prejuicios que anteriormente beneficiaban 


\section{Revista Iberoamericana \\ de las Ciencias Sociales y \\ Humanísticas}

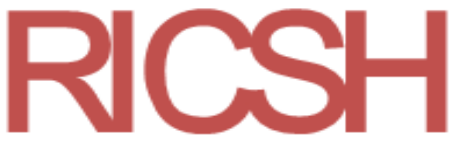

ISSN: $2395-7972$

al hombre y a la mujer sometían a esta última a los cánones del hogar y rigurosamente a la actividad reproductiva (lo privado) y no a la productiva (lo público).

En este mismo sentido, Flores (2014) retoma a Beauvoir y señala que la diferencia social entre los sexos tiene varias premisas:

La reproducción (biológica) es identificada como el fenómeno de sujeción al cual las mujeres han anclado su historia, de tal forma que el rol femenino fue determinado por la función reproductiva, ocasionando la exclusión de las mujeres de diferentes actividades que progresivamente se convirtieron en las más valoradas socialmente.

Así, la división del trabajo en función del sexo, significó la valoración de la diferencia de las tareas, que se convirtió gradualmente en inequidad entre los sexos (p. 17).

Ahí mismo se define al género "como un sistema que orienta las diferentes representaciones del sexo en función de exigencias culturales" (Flores, 2014, p. 20). Aún más, el género es un sistema ideológico cuyos diferentes métodos sitúan el modelaje de la representación social específica de los sexos; determina formas específicas de conducta establecidas en función del sexo biológico, y como un sistema de regulación social que orienta una estructuración cognitiva-social específica, que es construida a partir de lo biológico que normativiza las nociones de lo que "es" lo masculino y femenino. Esto significa que "la naturaleza asigna el 'dato biológico', pero la cultura es la que define las modalidades que la diferencia adopta" (Flores, 2014, p. 20). Es así como se han creado, objetivado y anclado las representaciones sociales de ser hombre y mujer y los roles asignados en una sociedad en específica.

Por su parte, Lamas (1995) menciona que el género es el conjunto de creencias, comportamientos, obligaciones, pero también derechos que se construyen socialmente tomando a la diferencia sexual como base. Este constructo social funciona como una especie de "filtro" cultural con el cual se explica al mundo, la forma en que se precisan las decisiones y oportunidades de las personas dependiendo de si tienen cuerpo de mujer o cuerpo de hombre. Las sociedades en el mundo clasifican cómo debe ser "lo propio" de las mujeres y "lo propio" de los hombres. Desde esas ideas culturales se establecen las obligaciones sociales de cada sexo con una serie de prohibiciones simbólicas, pero a su vez también de permisiones simbólicas. 

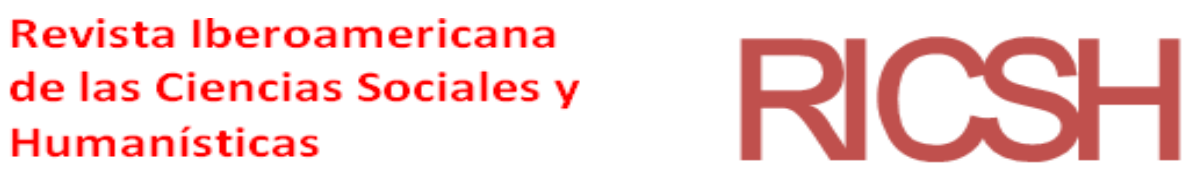

ISSN: $2395-7972$

Sin embargo, a diferencia del hombre, la mujer tiene algunas variantes culturales que pueden restringir la situación expuesta anteriormente. Lamas (1995) sugiere que en las sociedades se establecen obligaciones sociales que construyen barreras o limitantes que impiden que la mujer acceda a prevenciones sobre su salud.

El concepto de vulnerabilidad, según lo señalado por la Comisión Económica para América Latina y el Caribe [Cepal] (2002), refiere al resultado de la exposición a riesgos más la incapacidad para enfrentarlos y la inhabilidad para adaptarse activamente (p. 3).

Existen vulnerabilidades diferenciadas para hombres y mujeres, muchas de las cuales son consecuencia del proceso por el cual la sociedad dicta diferentes pautas de comportamiento a cada uno de los géneros, como se verá más adelante. Aquí, sin embargo, hablaremos específicamente de aquellas vulnerabilidades que afectan las mujeres, ya que consideramos importante, además de hacer visible su especificidad, poder identificar algunas de las determinantes de estas vulnerabilidades asociadas al hecho de pertenecer al sexo y al género femeninos. La vulnerabilidad de las mujeres tiene múltiples rostros: biológico, epidemiológico, social y cultural (Herrera y Campero, 2002, p. 5).

Tabla 1. Relación con el agresor

7 de cada 19 agresiones son cometidas por el esposo o pareja de la víctima.

En 2 de cada 10 casos el agresor es algún miembro de la familia.

1 de cada 10 ataques de género es cometido por una persona desconocida.

Fuente: Fiscalía Especializada de la Mujer Chihuahua (2020)

Las relaciones de poder en cuestiones de violencia de género en nuestra cultura han tenido prevalencia para que se sigan teniendo cifras altas en comportamientos de delitos.

Alonso y Castellanos (2006) mencionan que la violencia de género incluye toda la violencia contra las mujeres, no solo la violencia contra las mujeres en el ámbito familiar, una de las expresiones más frecuentes de la violencia de género. En la pareja no solo se produce violencia contra las mujeres, sino también violencia contra la pareja masculina y también podemos encontrar violencia entre las parejas del mismo sexo. Así pues, la violencia hacia la mujer en la pareja es uno de los subtipos de violencia de género.

Las relaciones de poder asimétricas que predominan en las parejas favorecen la sumisión y dependencia económica de las mujeres a los hombres, lo que con frecuencia 


\section{Revista Iberoamericana \\ de las Ciencias Sociales y \\ Humanísticas}

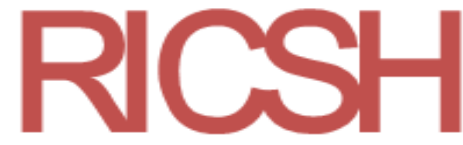

ISSN : 2395 - 7972

limita o provoca el no tener acceso a otra opción más que sufrir o soportar los diferentes tipos de violencias de género (Alonso y Castellanos, 2006).

A continuación, se muestra una gráfica sobre el comportamiento de delitos en el estado de Chihuahua.

Figura 2. Comportamiento de delitos

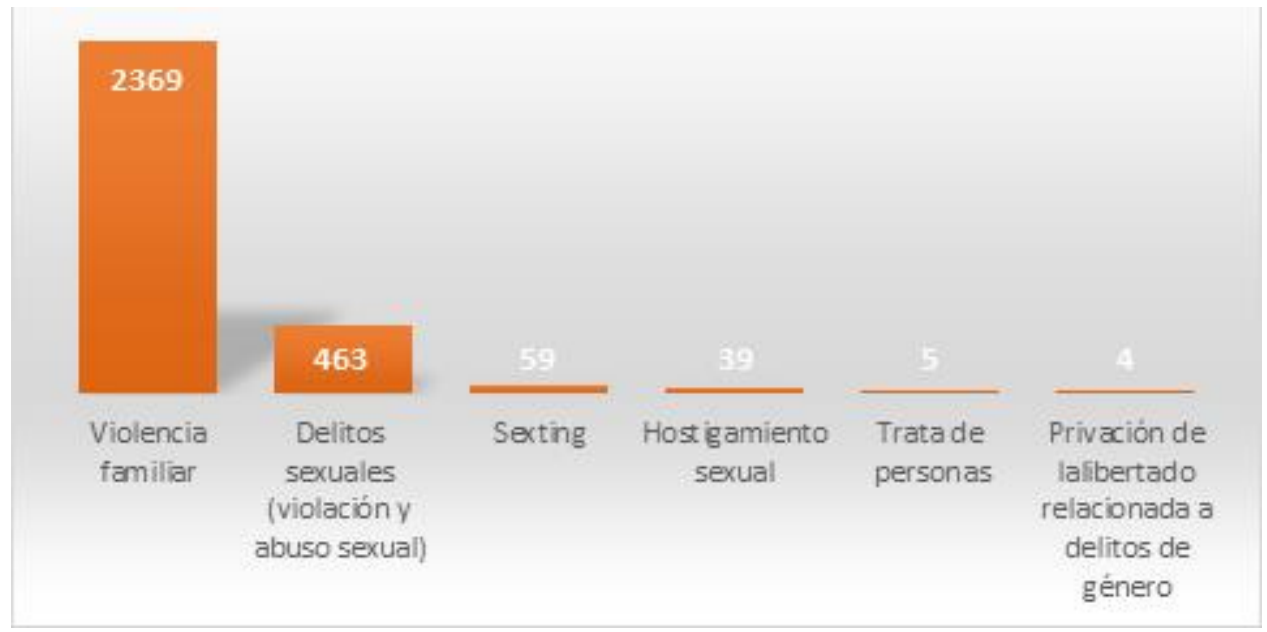

Fuente: Fiscalía Especializada de la Mujer Chihuahua (2020)

En relación con los conceptos de violencia de género y violencia feminicida, nos parece necesario hacer una descripción del marco referencial de este crimen (la violencia) para tener en cuenta la dimensión y comprender el objetivo de la intervención del Protocolo Policial de Atención a Mujeres Víctimas de Violencia de Género [en adelante Protocolo Policial] (Presidencia Municipal Juárez, Chihuahua, 2017) como una medida que permita prevenir y combatir las diferencias y dominaciones que las construcciones de género provocan.

Al pasar la violencia de género a formar parte de la agenda política internacional y nacional como un problema significativo por resolver, se ha generado un particular registro discursivo contenido en gran cantidad de declaraciones y convenciones (Organización de las Naciones Unidas [ONU], Organización de los Estados Americanos [OEA] y Unión Europea [UE], entre otras entidades internacionales), así como de medidas gubernamentales y parlamentarias. De hecho, ha sido definida por la ONU como "el crimen contra la humanidad más extendido, tolerado e impune y la primera causa de muerte o invalidez de mujeres entre 15 y 44 años en todo el mundo" (Torres y Antón, 2010, citados en González, 2012, p. 32).

En México, la violencia de género se conceptualiza como la violencia física o psicológica ejercida contra las mujeres por el hecho de ser mujeres. Según datos del censo realizado por el Instituto Nacional de Estadística y Geografía (Inegi) en 2015, 
Revista Iberoamericana

de las Ciencias Sociales y

Humanísticas

ISSN: $2395-7972$

en México habitaban 119938473 personas, de las cuales 51.4 \% eran mujeres (Pérez, 2016)

Ahora bien, de acuerdo con la Encuesta Nacional sobre Dinámica de las Relaciones en los Hogares (Endireh) 2016, 66.1\% de las mujeres mayores de 18 años habían sufrido algún tipo de agresión física, psicológica o sexual; es decir, 66 de cada 100 mujeres. Y solo $9.45 \%$ realizó una denuncia al respecto. La ONU clasifica al país entre las 20 peores naciones con problemas de dicha naturaleza, lo que lo convierte a este y a Centroamérica en la región del mundo más violenta para las mujeres fuera de una zona de guerra, según señala la Redacción Animal Político (22 de noviembre de 2017).

La violencia contra las mujeres se realizó principalmente en los ámbitos escolar (25.3\%), laboral (27\%) y comunitario (38.7\%), a través de acoso y abuso sexual (frases ofensivas, miradas lascivas, amenazas de violación, manoseos o exhibicionismo), hostigamiento, humillación e intimidación, acecho (ser seguidas en la calle), discriminación por embarazo, golpes y asesinatos, por mencionar algunas (Inegi, 2016)

La violencia contra las mujeres sigue en aumento en los últimos años. En 2019 fueron asesinadas 2173 mujeres. Según datos del Secretariado Ejecutivo del Sistema Nacional de Seguridad Pública [SESNSP] (25 de agosto de 2019), de enero a julio de 2019 se registraron 563 feminicidios y hubo 1610 mujeres víctimas de homicidio doloso. Además, siguiendo las estadísticas de este órgano administrativo, en el mes de abril del 2019, Ciudad Juárez ocupó el primer lugar de feminicidios a nivel nacional. En el mes de junio del mismo año, este municipio ocupó el primer lugar con 12 feminicidios.

De acuerdo con cifras oficiales de la organización Red Mesa de Mujeres, (Gamboa, 2019), con 100 mujeres asesinadas de enero a julio del 2019, Ciudad Juárez se convirtió en uno de los municipios del país con más feminicidios registrados. Cifras oficiales de Red Mesa de Mujeres detallan que en el mes de junio de ese año fueron 16 las mujeres asesinadas, en mayo y abril 15, en marzo 10, en febrero nueve y en enero 15. El grupo con más mujeres asesinadas es el que corresponde al rango de los 15 a 29 años, con 29 víctimas; después el de 30 a 50 años, con 26 mujeres asesinadas, y seis menores a 14 años habían sido asesinadas a mitad del 2019. En cuanto a las causas, se dio a conocer que 59 casos las mujeres fueron asesinadas con arma de fuego, 14 con algún objeto punzocortante, nueve por golpes y cinco por asfixia. Del resto, se desconoce hasta el momento la causa de muerte. Las zonas de la localidad con mayor incidencia son el Granjero, Felipe Ángeles, Senderos de San Isidro, Altavista, Anáhuac, Granjas de Chapultepec, La Cuesta, Manuel J. Clouthier, Obrera, Sierra Vista y Tierra Nueva. 
El problema de la violencia contra las mujeres ha sido uno que ha perdurado al interior de la sociedad mexicana; no fue hasta los últimos años, sin embargo, que el discernimiento de la violencia ejercida contra las mujeres ha cambiado radicalmente. Este escenario se da sobre todo a partir de que la violencia contra las mujeres pasó del ámbito privado para convertirse en un problema de la esfera pública. Esta nueva perspectiva ha sido esencial para entender las acciones que se han tomado por parte tanto de los Estados como de los organismos internacionales y de las organizaciones de la sociedad civil, de ahí que haya más participación en cuanto a iniciativas que busquen mejorar el combate y prevención del delito y la violencia, a propósito del Concurso Nacional de Mejores Prácticas Policiales basadas en la Evidencia (Pérez, 2016).

Los siguientes párrafos toman como fuente de información el material publicado por la Secretaría de Gobernación (Segob), el Instituto Nacional de las Mujeres (Inmujeres) y ONU Mujeres, La violencia feminicida en México, aproximaciones y tendencias 1985-2016 (Segob, Inmujeres y ONU Mujeres, 2017).

Dicho trabajo:

Constituye una actualización de dos publicaciones previas realizadas por la Entidad de las Naciones Unidas para la Igualdad de Género y el Empoderamiento de las Mujeres, ONU Mujeres, la Comisión Especial para el Seguimiento de los Feminicidios y el Inmujeres: Feminicidio en México: aproximación, tendencias y cambios 1985-2009 y Violencia feminicida en México. Características, tendencias y nuevas expresiones en las entidades federativas, 1985-2010, estas publicaciones han tenido el aporte técnico de El Colegio de México y en esta nueva edición se suma la Segob, la cual preside el Sistema Nacional de Prevención, Atención, Sanción y Erradicación de la Violencia contra las Mujeres (Segob, Inmujeres y ONU Mujeres, 2017, p. 10)

Se considera relevante mostrar las cifras y tendencias expuestas en dicho estudio a fin de esquematizar y resaltar la importancia del papel que deben tomar las instituciones públicas para centrarse en interrumpir este proceso de victimización e impedir futuros episodios violentos. "[Es una] necesidad (...) que el Estado y sus agentes intervengan más activamente en la prevención y la erradicación de la violencia contra las mujeres” (p. 12). También es responsabilidad del Estado y de sus servidores públicos el "asegurar y garantizar el ejercicio de los derechos humanos de las mujeres y en particular el derecho de éstas a una vida libre de violencia" (p. 12). Y distingue "distintos tipos de violencia (física, sexual y psicológica) y ámbitos de ocurrencia (familia, el lugar de trabajo, el 


\section{Revista Iberoamericana \\ de las Ciencias Sociales y \\ Humanísticas}

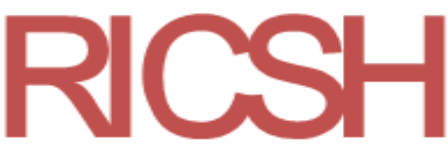

ISSN: $2395-7972$

ámbito escolar y el comunitario); tipos y ámbitos que deben ser atendidos por las políticas públicas" (p. 12).

Es de vital significación evitar que las conductas violentas sean denunciadas solo después de múltiples episodios de maltrato y abuso en sus distintas modalidades y que la violencia llegue hasta tal punto de su expresión última: el feminicidio, por lo que insistimos en la relevancia de contar con acciones por parte de las instituciones policiales que se especialicen en la adecuada atención de la violencia de género, a fin de que el servicio que otorguen combata y prevenga con eficiencia y eficacia esta situación social.

En el análisis de la investigación citada se definen los términos femicidio/ feminicidio según el Modelo de protocolo latinoamericano de investigación de las muertes violentas de mujeres por razones de género:

La muerte violenta de mujeres por razones de género, ya sea que tenga lugar dentro de la familia, unidad doméstica o en cualquier otra relación interpersonal, en la comunidad, por parte de cualquier persona, o que sea perpetrada o tolerada por el Estado y sus agentes, por acción u omisión (p. 12).

Lo anterior hace referencia indirectamente a la tipificación del feminicidio como delito autónomo en el Código Penal Federal mexicano (Segob, 24 de enero de 2020):

Comete el delito de feminicidio quien prive de la vida a una mujer por razones de género. Se considera que existen razones de género cuando concurra alguna de las siguientes circunstancias:

I. La víctima presente signos de violencia sexual de cualquier tipo;

II. A la víctima se le hayan infligido lesiones o mutilaciones infamantes o degradantes, previas o posteriores a la privación de la vida o actos de necrofilia;

III. Existan antecedentes o datos de cualquier tipo de violencia en el ámbito familiar, laboral o escolar, del sujeto activo en contra de la víctima;

IV. Haya existido entre el activo y la víctima una relación sentimental, afectiva o de confianza;

V. Existan datos que establezcan que hubo amenazas relacionadas con el hecho delictuoso, acoso o lesiones del sujeto activo en contra de la víctima; 
VI. La víctima haya sido incomunicada, cualquiera que sea el tiempo previo a la privación de la vida; VII. El cuerpo de la víctima sea expuesto o exhibido en un lugar público";

VII. El cuerpo de la víctima sea expuesto o exhibido en un lugar público. $^{2}$

\section{Discusión}

El presente apartado detalla la metodología para la elaboración del Protocolo Policial, cuya información ha sido tomada directamente del documento legal que autoriza la expedición del protocolo mismo (Presidencia Municipal Juárez, Chihuahua, 2017).

Las instituciones que coordinaron el proceso de elaboración del Protocolo Policial fueron la Dirección de Previsión Social de la SSPM, el IMM y el National Democratic Institute for International Affairs (NDI). La redacción del Protocolo Policial quedó a cargo del Instituto para la Seguridad y la Democracia (Insyde) y el NDI.

Para la realización del Protocolo Policial se consideró importante generar un espacio de diálogo en el que pudieran confluir las diferentes visiones vinculadas a las problemáticas de la violencia de género y la seguridad. Para ello, se realizaron mesas de trabajo en las que participaron servidores y servidoras públicas federales, estatales y municipales policías de la Unidad Especializada en Violencia Doméstica de la Policía Municipal de Ciudad Juárez (Unevid), representantes de las principales organizaciones de la sociedad civil y el sector académico de Ciudad Juárez que trabaja en la temática. Entre los meses de abril y junio de 2016 se organizaron cinco mesas de trabajo en las que, mediante una metodología de trabajo basada en la escucha activa y toma de notas sujetas a revisión y reelaboración crítica, se fueron generando, a través de la discusión argumentativa entre las y los actores participantes, los consensos respecto a los principales puntos a incluir en el Protocolo Policial (Presidencia Municipal Juárez, Chihuahua, 2017).

El objetivo general del Protocolo Policial es establecer criterios homólogos y eficientes que guíen la actuación de las y los policías del municipio de Juárez en la atención de casos de violencia de género contra las mujeres, con la finalidad de

\footnotetext{
${ }^{2}$ Artículo 325 del Código Penal Federal, última reforma publicada en el Diario Oficial de la Federación (DOF). Actualmente, los 32 códigos penales de las entidades federativas (CPEF) del país han tipificado el delito de feminicidio. Cabe destacar que no todas las definiciones en los CPEF están armonizadas con el Código Penal Federal; en este sentido, es necesaria su revisión y armonización en todo el territorio nacional.
} 


\section{Revista Iberoamericana \\ de las Ciencias Sociales y \\ Humanísticas}

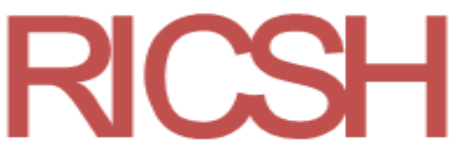

ISSN: $2395-7972$

estandarizar esta práctica policial en el contexto del sistema penal acusatorio y el nuevo régimen constitucional de derechos humanos.

Además, se establece que la función policial de atención a mujeres víctimas de violencia de género consiste en hacer cesar el riesgo o daño que sufren las víctimas, deteniendo materialmente la conducta violenta que les causa daño. Dicha función involucra un conjunto de movimientos que buscan hacer cesar cualquier conducta violenta de parte de una persona agresora mediante la aplicación inmediata de un amplio rango de fuerza que se extiende desde la mera presencia (con la presión psicológica que esta implica) hasta la fuerza física en sus diversos grados (Presidencia Municipal Juárez, Chihuahua, 2017).

El Protocolo Policial cuenta con tres principios transversales de operación en la atención a mujeres víctimas de violencia de género. El primero de ellos es el principio propersona, que se orienta a privilegiar, seleccionar y favorecer la aplicación de la norma jurídica que mejor proteja los derechos fundamentales del ser humano.

En tal sentido, la norma que prevalecerá es aquella que mejor proteja o menos restrinja al ser humano en el ejercicio de sus derechos fundamentales (...), pues lo importante es asegurar el ejercicio y garantía de los derechos humanos, preservar la dignidad y alentar el desarrollo de las personas (Presidencia Municipal Juárez, Chihuahua, 2017, p. 2).

El segundo principio es la perspectiva de género. Esta es entendida como una herramienta que identifica y reconoce las diferencias sociales entre mujeres, hombres y personas intersexuales para establecer acciones tendientes a promover situaciones de equidad. Al incorporar este enfoque, se puede identificar, cuestionar y valorar la discriminación, desigualdad y exclusión de las mujeres, que tradicionalmente se ha justificado por las diferencias biológicas.

También permite evaluar los impactos diferenciados de la aplicación concreta de la ley entre hombres y mujeres, dada la asignación de roles sociales en virtud del sexo, estereotipos sociales, patrones de discriminación y violencia ejercida y padecida por las mujeres en razón del género (Presidencia Municipal Juárez, Chihuahua, 2017, p. 2).

El tercer principio es la transparencia policial:

Por el cual se promueve el desarrollo de una gestión policial abierta y sometida al escrutinio público (...). La actividad policial debe ser pública y sometida al escrutinio de la población, y estar disponible a la 

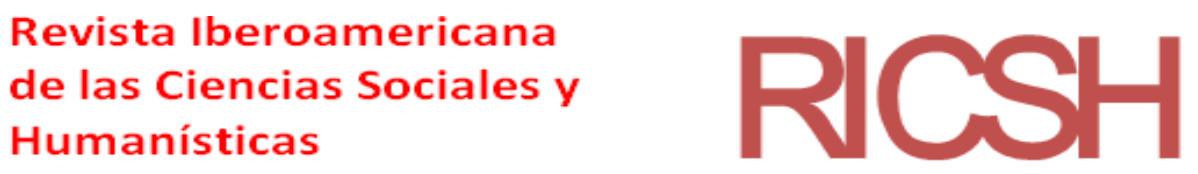

ISSN: 2395 - 7972

observación, monitoreo y evaluación ciudadana (Presidencia Municipal Juárez, Chihuahua, 2017, p. 2).

Estos tres principios son los que rigen toda la actuación policial en la atención a mujeres víctimas de violencia de género y deben ser tenidos en cuenta tanto en situaciones ordinarias como extraordinarias.

El Protocolo Policial establece cuatro fases en el proceso de atención: planeación, ejecución, custodia y traslado y comunicación. En cada una de ellas se establecen las acciones que se dirigen a la detección, identificación, intervención, atención, protección y prevención de situaciones o comportamientos violentos contra las mujeres por razones de género. Estas fases marcan procedimientos que brindan certeza jurídica a los y las policías y garantizan un accionar adecuado.

Consideramos necesario mencionar que la etapa de ejecución del proceso de atención a mujeres víctimas de violencia de género correspondiente a la intervención está disponible en el artículo 34 del protocolo en cuestión (Presidencia Municipal Juárez, Chihuahua, 2017).

Ahora bien, es necesario destacar la intervención de la Unevid a fin de ilustrar las acciones que tiene con la víctima como unidad encargada responsable en la primera interacción con esta.

- El personal operativo de la Unevid capacitado en la atención con perspectiva de género, cuyas intervenciones están apegadas al manual de procedimientos de la policía, realiza patrullaje preventivo y dan atención de los llamados al 911 en los diferentes distritos, atendiendo la mayor cantidad de folios de violencia familiar, omisión de cuidados, violaciones, abusos sexual y acoso, con el fin de que cada unidad de los distritos pueda atender diferentes estrategias preventivas que afectan en su sector. Además, se realizan apoyos a los diferentes juzgados familiares en la recuperación de menores, separación de personas, depósito de personas y notificaciones.

- Dentro de los folios atendidos, también se traslada a las víctimas a las diferentes instituciones con las que se está en coordinación para dar atención a sus denuncias cuando no existe flagrancia para una puesta a disposición o para su atención médica o psicológica (Presidencia Municipal Juárez, Chihuahua, 2017).

De acuerdo con Corchado (citada en Miranda, 21 de mayo de 2020), desde las medidas sanitarias implementadas por el Gobierno federal y estatal, a través de las cuales restringieron la actividad económica, tanto los padres y madres de familia se confinaron en sus casas y dejaron sus centros de trabajo, algunos de ellos con un porcentaje menor de sueldo por el cierre de la industria, empresas y comercio en la ciudad. 


\section{Revista Iberoamericana \\ de las Ciencias Sociales y \\ Humanísticas}

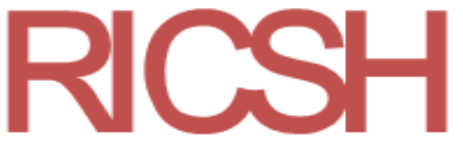

ISSN: $2395-7972$

Ante esta situación, la tensión y estrés de los integrantes de la familia fueron detonantes de actos de violencia familiar, antes los cuales, por lo regular, la víctima (mujer) tiene temor de denunciar, porque todo el tiempo está con ellos el agresor, coincidió la directora del IMM, Verónica Corchado Espinoza.

Me parece que en este confinamiento como están ahí generalmente los agresores, eso por un lado y por otro las mujeres están tan agobiadas con el trabajo o de la escuela que se les multiplica ahora como profesoras y también por el mismo trabajo de la casa que tienen hijos y lo que hace es que agrava la situación de violencia (Corchado, citada en Miranda, 21 de mayo de 2020, párr. 6).

Además, se agrega que, si los juzgados están cerrados, las víctimas no tienen otra manera de enfrentar esta situación. Por ello, se ha abierto diálogos con mujeres tanto a nivel privado como con grupos de cinco mujeres, asesorías virtuales que ayudan mucho a enfrentar este momento y darles apoyo, además de monitorear con algunas mujeres la situación que está prevaleciendo en sus casas.

Corchado Espinoza (citada en Miranda, 21 de mayo de 2020) informó que, respecto al año pasado, tienen un incremento de $15 \%$ en este año de casos que atendió la dependencia municipal en cuanto a violencia doméstica.

Ella misma indicó que una de las razones por lo que las mujeres no toman la decisión de denunciar una agresión es porque el agresor está permanentemente con ella; otra de las causas es la triple jornada, que trae como consecuencia que las mujeres queden invalidadas para tomar decisiones, y una tercera razón es que las condiciones no están dadas en la ciudad para salir.

La coordinadora del Centro de Justicia para las Mujeres del Gobierno del Estado (Cejum) afirmó, según la nota de Miranda (21 de mayo de 2020), que apoyan a las mujeres víctimas de violencia para interponer denuncia legal.

Se expuso que existe una coordinación también con la Unevid de la SSPM, esto es, cuando al momento de pedir ayuda no se consigue detener al agresor en el momento que suceden los hechos y se trasladan a los y las afectadas al Cejum para que interpongan su denuncia.

Por último, expresó que, desafortunadamente, por la cuestión de la contingencia, no hay manera de que las víctimas puedan salir con facilidad, porque su agresor se encuentra la mayor parte del tiempo junto a ellas.

Ante esta situación de inseguridad, las recomendaciones emitidas son que, si se diera el caso en que ellas consiguen hacerlo, lo primero que deben recordar es el pedir 
ayuda para poder darles el soporte que necesitan, puedan ser canalizadas a un refugio, darles el empoderamiento que precisan y evitar que regresen con el agresor (Corchado, 21 de mayo de 2020).

\section{Conclusiones}

Es de suma importancia que los Gobiernos municipales, estatales y federales tengan medidas para que esta violencia no se agrave, ya que debemos de tomar en cuenta que falta todavía la parte más crítica de esta contingencia. En varios países se han experimentado varios rebrotes y se debe de reconocer la amenaza de esta emergencia sanitaria: los Gobiernos tienen que proteger a los grupos vulnerables.

Finalmente, hay algunos puntos a tratar sobre este tema. El primero de ellos es mejorar la perspectiva de género en todas las respuestas que dé el Gobierno ante la contingencia del coronavirus, tomando en cuenta los distintos contextos que potencializan la violencia. También es imprescindible asegurar la participación de la mujer en posiciones de tomas de decisiones, así como el fortalecimiento de los servicios de respuesta a la violencia familiar y sexual en el contexto del confinamiento, es decir, se tienen que reformular los mecanismos tradicionales de respuesta ante una situación de violencia.

Es trascendental, igualmente, que se fortalezca la capacidad de los agentes de seguridad y actores de justicia, así como las sanciones por violencia familiar; ofrecer atención diferenciada (según su contexto real) a las mujeres que sufren algún tipo de violencia, garantizar la disponibilidad y continuidad de los servicios de salud sexual y reproductivo, así como la promoción de una política de salud mental.

Dentro de las futuras líneas de investigación queda pendiente abordar los efectos de la capacitación en el tema de la perspectiva de género en los funcionarios públicos, en especial en los policías municipales. Además, generar nuevas formas de intervención social de manera integral, es decir, desarrollar intervenciones sociales con mujeres y hombres. Este documento científico no alcanzó a abordar esos temas, primeramente por el tiempo, y en segundo lugar, porque se requiere que las diferentes áreas del municipio abran sus puertas para iniciar entrevistas para medir el impacto de las acciones que se han llevado con perspectiva de género, algo sumamente relevante para continuar desarrollando esta temática. 


\section{Futuras líneas de investigación}

Después del estudio realizado, se tiene claro que el confinamiento por la pandemia de Covid-19 aumentó el número de llamadas telefónicas por la violencia doméstica en el municipio de Ciudad Juárez, por lo que es urgente un protocolo de actuación que permita dar seguimiento a las llamadas al 911, se requiere una intervención multidisciplinaria, psicólogos, trabajadores sociales, sociólogos y médicos que den seguimiento de manera oportuna a los casos.

Lo que queda pendiente es conocer los factores que determinan la violencia de género en el ámbito doméstico mediante el diseño de escalas estadísticas con el interés de general políticas públicas que prevengan la violencia, sin embargo, se necesitan las condiciones adecuadas de salud para iniciar con estas nuevas líneas de investigación.

\section{Referencias}

Alonso, J. M. y Castellanos, J. L. (2006). Por un enfoque integral de la violencia familiar. Psychosocial Intervention, 15(3), 253-274.

Berger, S. (April 20, 2020). Gender-based violence in the COVID-19 pandemic. EurekAlert! Retrieved from https://www.eurekalert.org/pub_releases/202004/cums-gvi042020.php.

Buchholz, Y. (22 de abril de 2020). COVID-19: Incremento de la violencia de género, Investigación y ciencia. Investigación y Ciencia. Recuperado de https://www.investigacionyciencia.es/noticias/covid-19-incremento-de-laviolencia-de-gnero-18549.

Comisión Económica para América Latina y el Caribe [Cepal]. (2002). Vulnerabilidad sociodemográfica: viejos y nuevos riesgos para comunidades, hogares y personas. Santiago, Chile: Comisión Económica para América Latina y el Caribe.

Crean unidad para atender casos de violencia doméstica. (2 de abril de 2013). El Diario.mx. Recuperado de http://diario.mx/Local/2013-04-02_12847e29/creanunidad-para-atender-casos-de-violencia-domestica/.

Flores, F. (2014). Psicología social y género. El sexo como objeto de representación social (2. ${ }^{\mathrm{a}}$ ed.). Mérida, México: UNAM.

Gamboa, P. (30 de julio de 2019). Otra vez Juárez va a la cabeza en feminicidios. El Heraldo de Juárez. Recuperado de 
https://www.periodicoelmexicano.com.mx/policiaca/otra-vez-juarez-va-a-lacabeza-en-feminicidios-3967901.html.

González, M. (2012). La violencia contra las mujeres: análisis de las políticas públicas españolas desde la perspectiva de género. (tesis de doctorado). Universidad de Oviedo, Oviedo. http://digibuo.uniovi.es/dspace/bitstream/10651/30762/2/TD_MariaRosarioGonz alezArias.pdf.

Guba, E. y Lincoln, Y. (2000). Paradigmas en competencia en la investigación cualitativa. En Denman, C. y Haro, J. (comps.), Por los rincones. Antología de métodos cualitativos en la investigación social (1. ${ }^{\mathrm{a}}$ ed.) (pp. 113-145). Hermosillo, México: El Colegio de Sonora.

H. Ayuntamiento de Juárez. (3 de abril de 2020). Frente a la contingencia del COVID-19 el Gobierno Municipal sigue brindando atención a la violencia familiar. Sala de prensa. Recuperado de http://www.juarez.gob.mx/noticia/20386/frente-a-lacontingencia-del-covid-19-el-gobierno-municipal-sigue-brindando-atencin-a-laviolencia-familiar.

Hernández, R., Fernández, C. y Baptista, P. (2010). Metodología de la investigación. Ciudad de México, México: McGraw-Hill.

Herrera, C. y Campero, L. (2002). La vulnerabilidad e invisibilidad de las mujeres ante el VIH/SIDA: constantes y cambios en el tema. Salud Pública de México, 44(6).

Ilis, A. (1994). Técnicas de investigación bibliográfica. Caracas, Venezuela: Contexto Ediciones.

Instituto Nacional de Estadística y Geografía [Inegi]. (2016). Encuesta Nacional sobre la Dinámica de las Relaciones en los Hogares (Endireh) 2016. México: Instituto Nacional de Estadística y Geografía. Recuperado de https://www.inegi.org.mx/app/saladeprensa/noticia.html?id=3718.

King, G., Keohane, R. y Verna, S. (2000). El diseño de la investigación social. Madrid, España: Alianza.

Lamas, M. (1995). Usos, dificultades y posibilidades de la categoría género. La Ventana, (1), $10-61$. Recuperado de http://www.revistalaventana.cucsh.udg.mx/index.php/LV/article/view/2684.

Miranda, S. (21 de mayo de 2020). Bajaron en abril 8\% cifras de violencia familiar. El Heraldo de Juárez. Recuperado de https://www.elheraldodejuarez.com.mx/local/bajaron-en-abril-8-cifras-deviolencia-familiar-noticias-de-ciudad-juarez-5259167.html. 
Morales, O. (2003). Fundamentos de la investigación documental y la monografía. En Manual para la elaboración y presentación de la monografía (pp. 20-40). Perú: Universidad de Los Andes.

Morales, O. A. (2003). Fundamentos de la investigación documental y la monografía. En Espinoza, N. y Rincón, A. (eds.), Manual para la elaboración y presentación de la monografía. Mérida, Venezuela: Grupo Multidisciplinario de Investigación en Odontología, Facultad de Odontología, Universidad de Los Andes.Pérez, T. (2016). Diagnóstico sobre la violencia de género y social en la Ciudad de México. Ciudad de México, México: Instituto Nacional de Desarrollo Social Recuperado de

https://www.gob.mx/cms/uploads/attachment/file/159755/2010_OVSyG_DF_3_ CIPE.pdf.

Redacción Animal Político. (22 de noviembre de 2017). México encabeza situación crítica de violencia contra mujeres en América Latina: ONU. Animal Político. Recuperado de https://www.animalpolitico.com/2017/11/mexico-violenciamujeres-america-latina/.

Ritzer, G. (2003). Teoría sociológica contemporánea. Madrid, España: MacGraw-Hill.

Rodríguez, J. (2013). Paradigmas, enfoques y métodos en la investigación educativa. Investigación Educativa, 7(12), 23-40.

Secretaría de Gobernación [Segob]-Instituto Nacional de las Mujeres [Inmujeres]-ONU Mujeres. (2017). La violencia feminicida en México, aproximaciones y tendencias 1985-2016. México: Secretaría de Gobernación-Instituto Nacional de las MujeresONU Mujeres. Recuperado de https://mexico.unwomen.org/es/digiteca/publicaciones/2017/12/violenciafeminicida.

Presidencia Municipal Juárez, Chihuahua. (2017). Protocolo Policial de Atención a Mujeres Víctimas de Violencia de Género. Ciudad Juárez, Chihuahua: Presidencia Municipal Juárez, Chihuahua.

Secretaría de Gobernación. (24 de enero de 2020). Código Penal Federal. Diario Oficial de la Federación. Recuperado de https://mexico.justia.com/federales/codigos/codigo-penal-federal/gdoc/.

Secretariado Ejecutivo del Sistema Nacional de Seguridad Pública (SESNSP). (25 de agosto de 2019). Información sobre violencia contra las mujeres (Incidencia delictiva y llamadas de emergencia 9-1-1), julio 2019. México: Secretariado 
Revista Iberoamericana

de las Ciencias Sociales y

Humanísticas

ISSN: 2395 - 7972

Ejecutivo del Sistema Nacional de Seguridad Pública. Recuperado de https://www.gob.mx/sesnsp.

Tamayo, M. (2014). El proceso de la investigación científica. Ciudad de México, México: Limusa.

Torres , L. y Antón, E. (2006). Lo que Ud. debe saber sobre Violencia de Género. León, España: Caja España. 